\title{
Clinical profile and outcome of H1N1 influenza patients in a tertiary care hospital in Kochi, Kerala
}

\author{
Sethulakshmi Prasad, A. J. Indhu', R. Athish Peter Margos', Shoba Philip \\ Departments of Anaesthesia and Critical care and ${ }^{1}$ Critical care, Lourdes Hospital, Kochi, Kerala, India
}

\section{Abstract}

Background: After the 2009 pandemic, a re-emergence of hemagglutinin type 1 and neuraminidase type 1 (H1N1) influenza cases has been noted in India recently. The number of swab-positive cases has increased in the year 2017 compared to yesteryears. Since the current circulating strain (A/Michigan/7/2009 [H1N1] pdm09) is different from previous pandemic strains, a look into the clinical profile is imperative. Aims: The aim is to study the clinical, biochemical, and radiological profile of H1N1 patients at initial presentation and its influence on the mode of treatment and outcome. Patients and Methods: A cross-sectional record-based analysis of all confirmed cases of H1N1 influenza admitted at Lourdes Hospital, Kochi, Kerala, between 2015 and 2017. Confirmation of cases was done by reverse transcriptase polymerase chain reaction of respiratory specimens at Manipal Centre for Virus Research. Results: A total of 76 confirmed cases of H1N1 influenza were detected during the study period of which 36 required Intensive Care Unit admission. Most patients were between 51 and 60 years (25\%). The predominant presenting symptoms were fever (98.7\%), dry cough (61.8\%), breathlessness (53.9\%), and the most common auscultatory finding being bilateral crepitations (64.47\%). Around 32.89\% of cases presented with bilateral lung infiltrates on X-ray. Sixty-nine of 76 patients $(90.79 \%)$ survived the disease. Conclusions: Vaccination, early recognition of the disease, and prompt initiation of treatment seem to be the only way to reduce H1N1 disease progression and associated mortality. Patients with risk factors require additional attention as clinical course can be unpredictable. Pregnancy is associated with higher rate of complications. Early respiratory support helped in preventing progression to respiratory failure in most of our patients.

Keywords: Cross-sectional studies, hemagglutinin type 1 and neuraminidase type 1 subtype, India, influenza A virus, Intensive Care Units, reverse transcriptase polymerase chain reaction

\section{INTRODUCTION}

The hemagglutinin type 1 and neuraminidase type 1 (H1N1) virus popularly known as swine flu virus is a type of influenza A virus which resulted from a triple genetic re-assortment of human, avian, and swine influenza viruses..$^{[1,2]}$ The earliest cases were identified in Mexico and southern California in April 2009..$^{[1,2]}$ During the 2009 pandemic, there was an outbreak in India as well. A re-emergence of H1N1 influenza cases has been noted since 2015, and the number of cases continues to rise. The number of swab-positive cases has increased in the year 2017 compared to yesteryears. Since the current circulating strain (A/Michigan/7/2009 [H1N1] pdm09) is different from the previous pandemic strains, a look into the clinical profile seemed imperative.

\begin{tabular}{|l|l|}
\hline \multicolumn{2}{|c|}{ Access this article online } \\
\hline Quick Response Code: & Website: \\
\hline & www.ijrconline.org \\
\cline { 2 - 2 } & \\
\hline
\end{tabular}

We aimed to study the clinical, biochemical, and radiological profile of $\mathrm{H} 1 \mathrm{~N} 1$ patients at the time of initial presentation and its influence on the mode of treatment and outcome.

\section{Patients and Methods}

This was a cross-sectional record-based analysis of all the confirmed cases of H1N1 influenza admitted at Lourdes Hospital, Kochi, Kerala, between 2015 and 2017. Confirmation

Address for correspondence: Dr. Sethulakshmi Prasad, Department of Anaesthesia and Critical Care, Lourdes Hospital, Kochi, Kerala, India. E-mail: sethu2401@gmail.com

This is an open access journal, and articles are distributed under the terms of the Creative Commons Attribution-NonCommercial-ShareAlike 4.0 License, which allows others to remix, tweak, and build upon the work non-commercially, as long as appropriate credit is given and the new creations are licensed under the identical terms.

For reprints contact: reprints@medknow.com

How to cite this article: Prasad S, Indhu AJ, Margos RA, Philip S. Clinical profile and outcome of H1N1 influenza patients in a tertiary care hospital in Kochi, Kerala. Indian J Respir Care 2018;7:97-101. 
Prasad, et al.: Clinical profile and outcome of H1N1 influenza patients

of cases was done by reverse transcriptase polymerase chain reaction of respiratory specimens (throat/nasopharyngeal swab) at Manipal Centre for Virus Research. All patients who were found to be H1N1 throat or nasopharyngeal swab positive were included in the study and those who were found negative were excluded from the study.

Relevant history, clinical manifestations at presentation, investigations (complete blood count, renal function test, liver function test, chest X-ray, and arterial blood gas analysis), and outcome of all the cases were recorded. Treatment details including antiviral drugs (oseltamivir), requirement for ventilation, and use of other supportive measures were also recorded.

\section{RESULTS}

A total of 76 confirmed cases of H1N1 influenza detected during the period of 2015-2017 were included in the study. Majority of patients belonged to the age group of 51-60 years (19 patients $-25 \%$ ). However, all the age groups seem to be affected by the infection. Both genders were equally affected with slight female preponderance (55.36\% - females). Out of the 76 diagnosed cases, 36 patients required Intensive Care Unit (ICU) admission while rest were managed in ward.

The predominant symptoms at presentation were fever (98.7\%), dry cough $(61.8 \%)$, breathlessness $(53.9 \%)$, and productive cough (30.3\%). Other symptoms have been listed in Table 1.

Regarding time of presentation, only $11.84 \%$ of patients presented within $48 \mathrm{~h}$ of onset of symptoms. Majority of the patients $(46.05 \%)$ presented 5-10 days from the onset. Initiation of antiviral treatment, even when presenting late, was associated with improvement in outcome.

Most common comorbid conditions associated with H1N1 infection were diabetes mellitus (16\%), hypertension (16\%), chronic obstructive pulmonary disease (COPD) (11.84\%), pregnancy $(9.21 \%)$, and coronary artery disease $(7.89 \%)$. Other less commonly associated comorbidities are enumerated in Table 2. Out of these, pregnancy was associated with increased risk of complications and possibility of invasive ventilation.

Eighty percent of patients had baseline room air saturation between $91 \%$ and $100 \%$. Around $15.8 \%$ of patients presented with a room air saturation between $80 \%$ and $90 \%$ while $3.9 \%$ of patients presented with severe desaturation $(<80 \%)$. Patients who presented with low room air saturation required invasive ventilation and had rapid progression of disease.

Total leukocyte count (TLC) was normal in $49 \%$ of patients, decreased in $22.37 \%$, and increased in $13.16 \%$. Low TLC was more suggestive of H1N1 infection in the presence of appropriate clinical signs while higher TLC indicated secondary bacterial infection.

The most common auscultatory finding was bilateral crepitations $(64.47 \%)$. In most cases, the crepitations were initially restricted to lower lobe (heard best in the infrascapular area) then gradually progressing to upper zones. This reinforces the need to auscultate all areas of lung field on admission. Around $25 \%$ of cases had clear lung fields on admission. A small proportion of patients $(6.58 \%)$ also presented with only wheezing which was probably due to exacerbation of underlying obstructive lung disease. The auscultatory findings have been summarized in Figure 1.

Chest X-ray was normal in $47.37 \%$ of cases on admission. However, $32.89 \%$ of cases presented with acute respiratory distress syndrome (ARDS)-like picture on X-ray and 7.89\% of cases with both ARDS and pleural effusion. Around $10 \%-15 \%$ of patients who had a clear chest X-ray on admission developed infiltrates (predominantly in the lower zone) in the follow-up X-rays $48-72 \mathrm{~h}$ later. Hence, it is appropriate to obtain repeated X-rays in the presence of

\begin{tabular}{lc}
\hline Table 1: Symptoms & \\
\hline Symptoms & Number of patients (\%) \\
\hline Fever & $75(98.7)$ \\
Dry cough & $47(61.8)$ \\
Breathlessness & $41(53.9)$ \\
Productive cough & $23(30.3)$ \\
Sore throat & $14(18.4)$ \\
Coryza & $5(6.6)$ \\
H/O contact & $3(3.94)$ \\
H/O travel & $2(2.63)$ \\
\hline
\end{tabular}

\section{Table 2: Comorbidities}

\begin{tabular}{lc}
\hline Comorbidities & Number of patients (\%) \\
\hline Diabetes mellitus & $16(21.05)$ \\
Hypertension & $16(21.05)$ \\
COPD & $9(11.84)$ \\
Pregnancy & $7(9.21)$ \\
Coronary artery disease & $6(7.89)$ \\
Bronchial asthma & $5(6.57)$ \\
Immunosuppression & $3(3.95)$ \\
Neurological disorders & $3(3.95)$ \\
Chronic kidney disease & $2(2.63)$ \\
Chronic liver disease & $1(1.32)$ \\
Obesity & $1(1.32)$ \\
Obstructive sleep apnea & $1(1.32)$ \\
Psychiatric disorders & $1(1.32)$
\end{tabular}

COPD: Chronic obstructive pulmonary disease

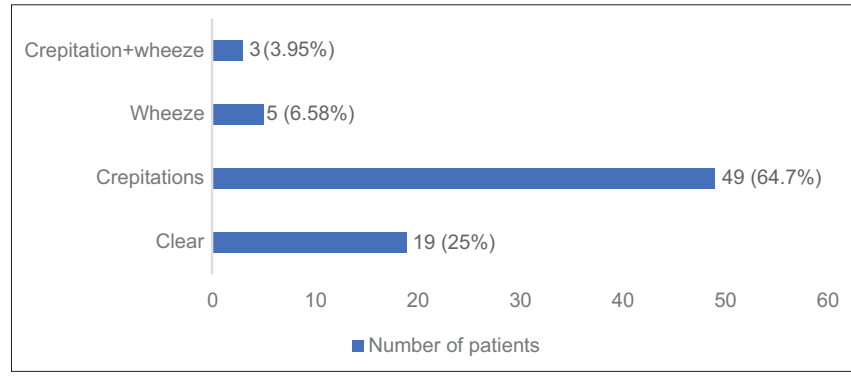

Figure 1: Auscultatory findings 
Prasad, et al.: Clinical profile and outcome of H1N1 influenza patients

correlating clinical findings. The chest X-ray findings have been summarized in Table 3.

Nearly $51.32 \%$ of cases did not require oxygen supplementation which mainly included patients admitted in ward. Patients admitted in ICU, however, required oxygen supplementation or ventilatory support in some form. About $21.05 \%$ of patients could be managed with noninvasive ventilation, but around $11.8 \%$ of patients required intubation and ventilation. Four of these patients who required invasive ventilation had to be tracheostomized due to prolonged ventilation. Most of these patients required an average 20-30 days of mechanical ventilation. This has been summarized in Table 4 .

The dose of oseltamivir dose used for treatment in $80 \%$ of adults included in the study was the prescribed dose of $75 \mathrm{mg}$ bd for 5 days. However, $20 \%$ of patients required an increase in the dose up to $150 \mathrm{mg}$ bd due to the severity of infection and rapid progression of the disease.

Of all the patients, 69 (90.79\%) patients survived the disease while $4(5.26 \%)$ patients succumbed [Table 5].

Out of the four patients, one was pregnant which probably worsened the prognosis. She also developed myocardial dysfunction (probably due to viral myocarditis) and bilateral bronchopleural fistula. Two out of these four patients died due to refractory hypoxemia. Since other modes of maintaining oxygenation such as high-frequency oscillatory ventilation (HFOV) and extracorporeal membrane oxygenation (ECMO) were not affordable by the patients, they succumbed to severe hypoxia. Another death was due to multiorgan dysfunction. One of the pregnant patients who survived developed unilateral foot drop probably due to viral invasion of sciatic nerve.

\section{Discussion}

After control of the $2009 \mathrm{H} 1 \mathrm{~N} 1$ pandemic, the number of cases across the world has reduced. But unfortunately, India has seen a continuous rise in the number of H1N1-positive cases since 2015. Although the survival rates have improved compared to the pandemic period, the number of swab-positive cases continues to rise. A review of the statistics in India can give a gross idea about the picture. The year of 2016 saw 1786 swab-positive cases with 265 deaths. However, in 2017, 12,460 swab-positive cases have been detected till July 9, 2017. Out of these cases, 600 patients succumbed to the disease. While the numbers are definitely much larger than figures of 2016, the death rate seems to have reduced from $14.8 \%$ (2016) to $4 \%(2017) \cdot{ }^{[3]}$ Although the worst outbreak in India was in 2009-2010 (50,000 affected people and 2700 deaths), a re-emergence of H1N1 is equally alarming.

The management of influenza A/H1N1 cases in India requires classification of these patients as per the revised guidelines of Ministry of Health and Family Welfare, Government of India, into three categories - Category A includes mild cases where no laboratory testing or antiviral treatment is needed. Category B includes high-risk patients such as children, pregnant women, persons aged $\geq 65$ year, and people with chronic disease. These patients need not be laboratory confirmed but should be isolated at their homes and given oseltamivir treatment. Category $\mathrm{C}$ patients are severe cases who need hospitalization, laboratory testing, and oseltamivir treatment. ${ }^{[4]}$ Most of the cases admitted in our hospital were Category B or C.

Treatment of all the cases included antiviral medication and supportive management. The antiviral used in our center is neuraminidase inhibitor, oseltamivir, which acts by inhibiting the aggregation and release of viral particles from infected cells. The treatment recommendations for oseltamivir are given in Table 6.

Evidence indicates that oseltamivir, when properly prescribed, significantly decreases risk of pneumonia (a leading cause of death) and the need for hospitalization. Although the recommended treatment duration is 5 days, longer treatment regimens are recommended for severely ill hospitalized patients or persons with immunosuppression. The Indian Society of Critical Care Medicine recommends higher doses and longer treatment duration in patients with severe or progressive clinical illness. In adults, a dose of $150 \mathrm{mg}$ bd for 10 days is being used and higher doses have been used in

\begin{tabular}{lc}
\hline Table 3: Chest X-ray findings & \\
\hline Chest X-ray findings & Number of patients (\%) \\
\hline Normal & $36(47.37)$ \\
ARDS & $25(32.89)$ \\
Consolidation & $9(11.84)$ \\
ARDS + pleural effusion & $6(7.89)$ \\
\hline
\end{tabular}

Table 4: Ventilator requirement

\begin{tabular}{lc}
\hline Mode of ventilation & Number of patients (\%) \\
\hline Noninvasive & $16(21.05)$ \\
Invasive & $9(11.84)$ \\
Controlled oxygen therapy & $12(15.79)$ \\
Room air & $39(51.32)$ \\
\hline
\end{tabular}

\begin{tabular}{lc}
\hline Table 5: Outcome & \\
\hline Outcome & Number of patients (\%) \\
\hline Survived & $69(90.79)$ \\
Death & $4(5.26)$ \\
Discharge at request & $3(3.95)$ \\
\hline
\end{tabular}

\begin{tabular}{llc}
\hline \multicolumn{3}{l}{ Table 6: Treatment recommendations for oseltamivir } \\
\hline Antiviral agent & Purpose & Dosage in adults \\
\hline Oral oseltamivir & $\begin{array}{l}\text { Chemoprophylaxis (7 days - } \\
\text { CDC 10 days - ISCCM) }\end{array}$ & $75 \mathrm{mg}$ OD \\
Oral oseltamivir & Treatment (5 days) & $75 \mathrm{mg} \mathrm{BD}$ \\
\hline
\end{tabular}

CDC: Centre for disease control, ISCCM: Indian Society of Critical Care Medicine 
Prasad, et al.: Clinical profile and outcome of H1N1 influenza patients

ICU patients. ${ }^{[5]}$ However, reduction to $75 \mathrm{mg}$ od is required for creatinine clearance $<30 \mathrm{ml} / \mathrm{min} \cdot{ }^{[6]}$ Insufficient safety data are available for doses $>75 \mathrm{mg}$ bd in pregnancy.

The most common presenting symptoms in our study were fever $(98.7 \%)$, cough $(61.8 \%)$, and progressive breathlessness $(41 \%)$. Although these findings are similar to the pandemic era of 2009-2010, incidence of sore throat was found to be low in our study (18.4\%). Studies during the pandemic period had reported high incidence of sore throat as presenting symptom. Chudasama et al. and Mehta et al. reported around $54 \%$ of cases presenting with sore throat. ${ }^{[7,8]}$ A study done by Jayadeva et al. also found myalgia common in their patients. ${ }^{[9]}$ Both genders were observed to be equally affected in our study similar to all the previous studies. ${ }^{[7-10]}$ Regarding associated comorbidities, we found diabetes mellitus $(21.05 \%)$, hypertension $(21.05 \%)$, COPD $(11.84 \%)$, and pregnancy $(9.21 \%)$ as the most common coexisting condition. Diabetes has been reported as a common coexisting condition in several previous studies. ${ }^{[7,8]}$ Kashinkunti et al. reported that around $45 \%-50 \%$ of their cases had diabetes mellitus or hypertension as a comorbidity. ${ }^{[1]}$ Chudasama et al. also commented on the fact that the common risk factors in India were found to be diabetes and hypertension while that in the US was bronchial asthma and COPD. ${ }^{[7]}$ Pregnancy has been reported as the most constant risk factor with high disease mortality. ${ }^{[12-14]}$ Our findings also correlated with the same. Although most previous studies recommend oseltamivir to be initiated within $48 \mathrm{~h}$ of onset for efficacy, we found that even when started after $48 \mathrm{~h}$, the clinical efficacy is maintained. Similar to the chest X-ray findings obtained by Kashinkunti et al. and Puvanalingam et al., our study also showed higher incidence of lower zone involvement with bilateral findings more common than unilateral. ${ }^{[11,13]}$ Initial presentation with bilateral lung infiltrates was associated with higher incidence of mechanical ventilation. Furthermore, early initiation of respiratory support in the form of noninvasive ventilation was found to be helpful in preventing progression to respiratory failure and reducing the requirement of invasive ventilation in most of our patients. Managing severe ARDS secondary to H1N1 influenza can be very difficult as has been described in a recent article by Pinto and Abayadeera. ${ }^{[15]}$ Such patients usually had a prolonged course in ICU and required tracheostomy. According to our findings, the most common causes of death were refractory hypoxemia and multiorgan dysfunction. Puvanalingam et al. also found pneumonia as the most common cause of death. ${ }^{[13]}$

A recent article by Murhekar and Mehendale on influenza A (H1N1) pdm09 outbreak in India suggested that there is no significant genetic change in the virus affecting its virulence. ${ }^{[16]}$ Even our findings from the present study suggest that the clinical profile appears to have remained constant from the pandemic era, but the mortality rate has definitely decreased probably owing to the early recognition of the disease and prompt initiation of treatment now. Surely, these numbers can be reduced further. Health education and vaccination are the promising tools to prevent further epidemics. An article by Mossad stated that the only way to combat the continuing pandemics was by vaccination. ${ }^{[17]}$ Although patients in high-risk group are definite candidates for vaccination, it should not be restricted to this group. Health-care personnel and persons coming in close contact with infected persons should also be advised to get vaccinated. The live attenuated vaccine has been found to be more effective and also helpful in providing herd immunity and hence should be preferred over inactivated vaccine.

\section{Conclusions}

Although the pandemic era of H1N1 has settled, epidemics are a constant reminder of the hidden danger. Vaccination, early recognition of the disease, and prompt initiation of treatment seem to be the only way to reduce the disease progression and associated mortality. Patients with risk factors require additional attention as clinical course can be unpredictable. Pregnancy deserves a special mention as both the mother and fetus are found to have a high morbidity and mortality.

\section{Recommendations}

- Regular vaccination against the most recent circulating strains can help in reducing the number of cases. Inactivated vaccine should be recommended to every pregnant lady considering the high mortality rate during pregnancy

- Creating awareness about the symptoms of the disease can help in early contact with medical facility and hence treatment

- Stable patients who do not require ICU care should be advised rest and cough etiquette.

\section{Limitations}

- Since this is a single center study, the results may not be representative of the general population

- Nonavaialbility of HFOV and ECMO at our center.

\section{Financial support and sponsorship}

Nil.

\section{Conflicts of interest}

There are no conflicts of interest.

\section{REFERENCES}

1. Baden LR, Drazen JM, Kritek PA, Curfman GD, Morrissey S, Campion EW, et al. H1N1 influenza a disease - Information for health professionals. N Engl J Med 2009;360:2666-7.

2. Belshe RB. Implications of the emergence of a novel H1 influenza virus. N Engl J Med 2009;360:2667-8.

3. Hindustan Times. Available from: http://www.hindustantimes.com/ health/alarming-rise-in-swine-flu-cases-in-india-as-345-killed-thisyear-alone/story-ykBIl6q7QT4ZJRZgNSRqaO.html. [Last accessed on 2018 Jan 18].

4. Ministry of Health and Family Welfare, Government of India. Guidelines on Categorization of Influenza A H1N1 cases During Screening for Home Isolation, Testing Treatment, and Hospitalization. Available from: http:// mohfw.gov.in/showfile.php?lid=3071. [Last accessed 2018 May 02]. 
5. Ray B, Samaddar DP, Todi SK, Ramakrishnan N, John G, Ramasubban S, et al. Quality indicators for ICU: ISCCM guidelines for ICUs in India. Indian J Crit Care Med 2009;13:173-206.

6. He G, Massarella J, Ward P. Clinical pharmacokinetics of the prodrug oseltamivir and its active metabolite ro 64-0802. Clin Pharmacokinet 1999;37:471-84

7. Chudasama RK, Patel UV, Verma PB, Amin CD, Savaria D, Ninama R, et al. Clinico-epidemiological features of the hospitalized patients with 2009 pandemic influenza A (H1N1) virus infection in Saurashtra region, India (September, 2009 to February, 2010). Lung India 2011;28:11-6.

8. Mehta AA, Kumar VA, Nair SG, K Joseph F, Kumar G, Singh SK, et al. Clinical profile of patients admitted with swine-origin influenza A (H1N1) virus infection: An experience from A tertiary care hospital. J Clin Diagn Res 2013;7:2227-30.

9. Jayadeva B, Chikkananjaiah N, Gowda V, Rani D, Poovathingal S. Clinical profile of suspected and confirmed H1N1 influenza infection in patients admitted at a tertiary care teaching hospital. J Cardiothorac Med 2015;3:367-70.

10. Amaravathi KS, Sakuntala P, Sudarsi B, Manohar S, Nagamani R, Rao SR. Clinical profile and outcome of recent outbreak of influenza a
H1N1 (swine flu) at a tertiary care center in Hyderabad, Telengana. Ann Trop Med Public Health 2015;8:267-71.

11. Kashinkunti MD, Gundikeri SK, Dhananjaya M. Study of clinical profile of patients with H1N1 influenza in a teaching hospital of North Karnataka. IJRRMS 2013;3:53-5.

12. Malhotra B, Singh R, Sharma P, Meena D, Gupta J, Atreya A, et al. Epidemiological and clinical profile of influenza A (H1N1) 2009 virus infections during 2015 epidemic in Rajasthan. Indian J Med Res 2016;144:918-23.

13. Puvanalingam A, Rajendiran C, Sivasubramanian K, Ragunanthanan S, Suresh S, Gopalakrishnan S, et al. Case series study of the clinical profile of H1N1 swine flu influenza. J Assoc Physicians India 2011;59:14-6, 18.

14. Agarwal S, Nawal CI, Agarwal A, Mittal P. Paripex Indian J Res 2018;7:39-41.

15. Pinto V, Abayadeera A. Are we ready to proceed with publications?. Sri Lankan J Anaesthesiol 2017;25:60-2.

16. Murhekar M, Mehendale S. The 2015 influenza A (H1N1) pdm09 outbreak in India. Indian J Med Res 2016;143:821-3.

17. Mossad SB. Influenza: Still more important than zika virus in 2016-2017. Cleve Clin J Med 2016;83:836-40. 